\title{
Global dynamics of Hořava-Lifshitz cosmology with non-zero curvature and a wide range of potentials
}

\author{
Fabao Gao ${ }^{1,2, \mathrm{a}_{\mathbb{D}}, \text { Jaume Llibre }^{2, \mathrm{~b}}}{ }_{(\mathbb{D})}$ \\ ${ }^{1}$ School of Mathematical Science, Yangzhou University, Yangzhou 225002, China \\ 2 Departament de Matemàtiques, Universitat Autònoma de Barcelona, Bellaterra, 08193 Barcelona, Catalonia, Spain
}

Received: 6 November 2019 / Accepted: 4 February 2020 / Published online: 17 February 2020

(C) The Author(s) 2020

\begin{abstract}
The global dynamics of a cosmological model based on Hořava-Lifshitz gravity in the presence of curvature is described by using the qualitative theory of differential equations.
\end{abstract}

\section{Introduction}

In recent years Hořava [1] proposed a spacetime asymmetric gravitational theory similar to Lifshitz's scalar field theory, also known as Hořava-Lifshitz gravity. This theory has inspired a great deal of research for its applications in cosmology and black hole physics (see [2-18] or the review articles $[19,20]$ and the references therein).

With or without detailed-balance condition, few researchers have published several papers on Hořava-Lifshitz gravity through phase space analysis, see for instance [3$7,17]$. Among these papers there are also some of them about the case of non-zero space curvature $k$. When the terms related to $k$ are dominant, Friedmann solution will be generated. On the contrary, when the effective Hořava radiation dominates, the corresponding cosmology gives a solution of "radiation-dominated like" [6]. According to whether the cosmological constant $\Lambda$ is zero in flat $(k=0)$ and non-flat $(k \neq 0)$ universe, Leon et al. [3-5,14] divided the HořavaLifshitz gravity into four cases: (1) $k=0, \Lambda=0$; (2) $k=0, \Lambda \neq 0$; (3) $k \neq 0, \Lambda=0$; (4) $k \neq 0, \Lambda \neq 0$, and analyzed these phase spaces. More precisely, they either studied the global dynamics of the planar case of HořavaLifshitz gravity with exponential potential by using the twodimensional Poincaré compactification, or discussed only local dynamics of this cosmological model with powerlaw potential but without investigate the dynamics close to infinity. The three-dimensional global dynamics of Hořava-

\footnotetext{
a e-mail: gaofabao@ sina.com (corresponding author)

be-mail: jllibre@mat.uab.cat
}

Lifshitz cosmology with $k=0$ and $\Lambda=0$ was studied in [7].

In this paper we will investigate the global dynamics of the Hořava-Lifshitz scalar field cosmology under the Friedmann-Lemaître-Robertson-Walker background spacetime in the presence of curvature and no cosmological constant term, i.e., $k \neq 0$ and $\Lambda=0$.

\section{The cosmological equations}

To describe the cosmological equations in this section we first give a brief review of the Hořava-Lifshitz gravitational theory as was proposed in [1]. In one common version of this theory [5,21], its field content can be given by a spatial scalar $N$, and a spatial vector $N_{i}$. They are the 'lapse' and 'shift' variables usually found in general relativity. Then the full metric can be written as

$$
\begin{aligned}
d s^{2} & =-N^{2} d t^{2}+g_{i j}\left(d x^{i}+N^{i} d t\right)\left(d x^{j}+N^{j} d t\right), \\
N_{i} & =g_{i j} N^{j}
\end{aligned}
$$

where $g_{i j}(i, j=1,2,3)$ is the spatial metric. The scaling transformation of the coordinates is in the form of $t \rightarrow l^{3} t, x^{i} \rightarrow l x^{i}$, under which both $N$ and $g_{i j}$ are invariant, but $N^{i}$ is scaled to $N^{i} \rightarrow l^{-2} N_{i}$.

Based on the detailed-balance condition [1], the full gravitational action of Hořava-Lifshitz is represented by

$$
\begin{aligned}
S_{g}= & \int d t d^{3} x \sqrt{g} N\left\{\frac{2}{\kappa^{2}}\left(K_{i j} K^{i j}-\lambda K^{2}\right)-\frac{\kappa^{2}}{2 w^{4}} C_{i j} C^{i j}\right. \\
& +\frac{\mu \kappa^{2}}{2 w^{2}} \frac{\epsilon^{i j m}}{\sqrt{g}} R_{i l} \nabla_{j} R_{k}^{l}-\frac{\mu^{2} \kappa^{2}}{8} R_{i j} R^{i j} \\
& \left.-\frac{\mu^{2} \kappa^{2}}{8(3 \lambda-1)}\left(\frac{1-4 \lambda}{4} R^{2}+\Lambda R-3 \Lambda^{2}\right)\right\},
\end{aligned}
$$


with the Cotton tensor $C^{i j}=\epsilon^{i j m} \nabla_{k}\left(4 R_{i}^{j}-R \delta_{i}^{j}\right) /(4 \sqrt{g})$ and the extrinsic curvature $K_{i j}=\left(\dot{g}_{i j}-\nabla_{i} N_{j}-\nabla_{j} N_{i}\right) /(2 N)$. The standard general covariant antisymmetric tensor is represented by $\epsilon^{i j m} / \sqrt{g}$, and the indices are to raise and lower with the metric $g_{i j}$. Besides $\lambda$ is a dimensionless constant, $\mu, \kappa$ and $w$ are also constants.

For the potential $V(\phi)$, we consider the gravitational action term as follows

$S=\int d t d^{3} x \sqrt{g} N\left(\frac{3 \lambda-1}{4} \frac{\dot{\phi}^{2}}{N^{2}}-V(\phi)\right)$,

and the metric $N^{i}=0, g_{i j}=a^{2}(t) \gamma_{i j}, \gamma_{i j} d x^{i} d x^{j}=$ $r^{2} d \Omega_{2}^{2}+d r^{2} /\left(1-k r^{2}\right)$, here $a(t)$ is the dimensionless scale factor for the expanding universe, $\gamma_{i j}$ is a constant curvature metric of maximally symmetric. The curvature $k=1,0,-1$ corresponding to the closed, flat, and open space, respectively.

For simplicity we normalize $\kappa^{2}$ and $N$ to the number one, then the cosmological equations can be described as

$$
\begin{gathered}
H^{2}=\frac{4 V(\phi)+(3 \lambda-1) \dot{\phi}^{2}}{24(3 \lambda-1)} \\
-\frac{\mu^{2} \Lambda^{2} a^{4}-2 \mu^{2} \Lambda k a^{2}+\mu^{2} k^{2}}{16 a^{4}(3 \lambda-1)^{2}}, \\
\dot{H}+\frac{3}{2} H^{2}=\frac{4 V(\phi)-(3 \lambda-1) \dot{\phi}^{2}}{16(3 \lambda-1)} \\
+\frac{3 \mu^{2} \Lambda^{2} a^{4}-2 \mu^{2} \Lambda k a^{2}+\mu^{2} k^{2}}{32 a^{4}(3 \lambda-1)^{2}}, \\
\ddot{\phi}+3 H \dot{\phi}+\frac{2 V^{\prime}(\phi)}{3 \lambda-1}=0,
\end{gathered}
$$

where $H=\dot{a} / a$ is the Hubble parameter.

Note that the cosmological constant term is neglected in the present paper, i.e., $\Lambda=0$, so system (4) reduces to

$$
\begin{aligned}
& H^{2}=\frac{\dot{\phi}^{2}}{24}+\frac{V(\phi)}{6(3 \lambda-1)}-\frac{\mu^{2} k^{2}}{16 a^{4}(3 \lambda-1)^{2}}, \\
& \dot{H}+\frac{\dot{\phi}^{2}}{8}=\frac{\mu^{2} k^{2}}{8 a^{4}(3 \lambda-1)^{2}}, \\
& \ddot{\phi}+3 H \dot{\phi}+\frac{2 V^{\prime}(\phi)}{3 \lambda-1}=0 .
\end{aligned}
$$

For simplicity the dimensionless transformation of the following variables $[4,5]$ is given as

$$
\begin{aligned}
& x=\frac{\dot{\phi}}{2 \sqrt{6} H}, \quad y=\frac{\sqrt{V(\phi)}}{\sqrt{6} H \sqrt{3 \lambda-1}}, \quad z=\frac{\mu}{4(3 \lambda-1) a^{2} H}, \\
& s=-\frac{V^{\prime}(\phi)}{V(\phi)}, \quad f(s) \equiv \frac{V^{\prime \prime}(\phi)}{V(\phi)}-\frac{V^{\prime}(\phi)^{2}}{V(\phi)^{2}} .
\end{aligned}
$$

Since the usual scalar field potential $V(\phi)$ admits multiple representations, the potential function $f(s)$ also has various mathematical expressions (see the papers [4, 17,22,23] and the references therein). In this paper we will investigate the monomial potential $V(\phi)=(\mu \phi)^{2 n} / 2 n$ with a positive constant $\mu$ and a natural number $n$. Thus the power-law potential is $f(s)=-s^{2} /(2 n)$, and we obtain $d s / d t=\sqrt{6} x s^{2} / n$. Furthermore it can be followed from the first three transformations in Eq. (6) and the first two equations in system (5) that $x^{2}+y^{2}-z^{2}=1$ and $3 x^{2}-2 z^{2}=-H^{\prime} / H$. Therefore the cosmological equations are reduced to the following autonomous dynamical system

$$
\begin{aligned}
& \frac{d x}{d t}=x\left(3 x^{2}-2 z^{2}-3\right)+\sqrt{6} s\left(1-x^{2}+z^{2}\right) \\
& \frac{d z}{d t}=z\left(3 x^{2}-2 z^{2}-2\right) \\
& \frac{d s}{d t}=\frac{\sqrt{6}}{n} x s^{2} .
\end{aligned}
$$

Our study will fully describes the global dynamics of system (7) in the region $G=\left\{(x, z, s) \in \mathbb{R}^{3}: x^{2}-z^{2} \leq 1\right\}$ of physical interest adding its boundary at infinity. It should be noted from the derivation of system (7) that the space curvature $k$ is implicit in the variables $x$ and $z$. More details of system (7) can be found from Eqs. (113)-(115) of [4] or Eqs. (44)-(46) of [14].

\section{Phase portraits on the invariant planes and surface}

In order to study the local phase portraits of the finite and infinite equilibrium points, and the global phase portraits of system (7) in the region $G$, which is the meaningful region for cosmology, see again [4] or [14]. We start discussing the phase portraits on its invariant planes and surface

$z=0, s=0, x^{2}-z^{2}=1$.

3.1 The invariant plane $z=0$

On this plane system (7) becomes

$\frac{d x}{d t}=\left(x^{2}-1\right)(3 x-\sqrt{6} s)$

$\frac{d s}{d t}=\frac{\sqrt{6}}{n} x s^{2}$.

The phase portraits of system (8) in the strip $z=0$ and $x^{2}-z^{2} \leq 1$, i.e., in $z=0,-1 \leq x \leq 1$, it has been studied in [7], and the phase portraits is shown in Fig. 1, where the hyperbolic equilibrium point $e_{0}=(0,0)$ is a saddle, both the semi-hyperbolic equilibrium points $e_{1}=(1,0)$ and $e_{2}=(-1,0)$ are saddle-nodes.

In order to describe the dark energy or dark matter in the Hořava-Lifshitz universe more clearly, we introduce the density parameters of dark energy and dark matter (see $[3,5]$ for more details): $\Omega_{D E}=-k^{2} z^{2}-u^{2}, \Omega_{M}=x^{2}+y^{2}$, as well as the corresponding equation-of-state parameters: $\omega_{D E}=$ $\left(k^{2} z^{2}-3 u^{2}\right) /\left(3 k^{2} z^{2}+3 u^{2}\right), \omega_{M}=\left(x^{2}-y^{2}\right) /\left(x^{2}+y^{2}\right)$. 

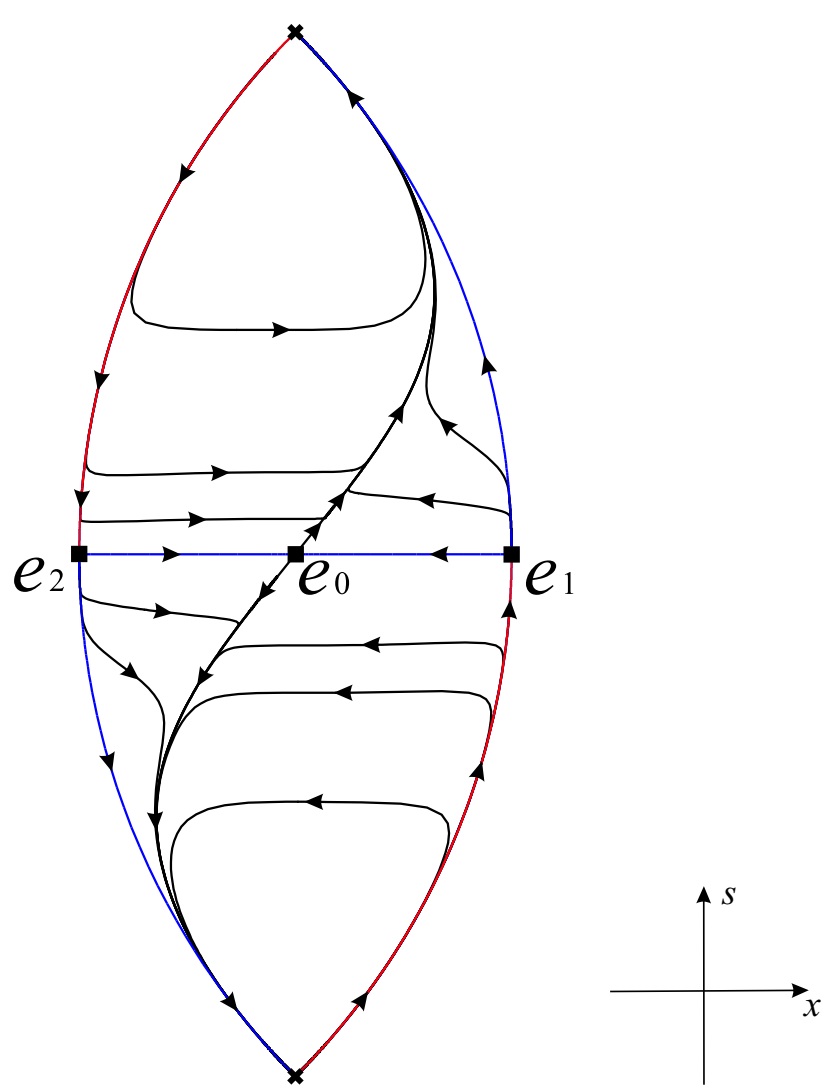

Fig. 1 The phase portraits of the invariant plane $z=0$ in the region $-1 \leq x \leq 1$

Table 1 Equilibrium points and their characteristics on the invariant plane $z=0$ for all $s \in \mathbb{R}$

\begin{tabular}{lcclcll}
\hline $\begin{array}{l}\text { Equilibrium } \\
\text { points }\end{array}$ & $x$ & $\mathrm{~s}$ & Stability & $\omega_{M}$ & $\Omega_{D E}$ & $\omega_{D E}$ \\
\hline$e_{0}$ & 0 & 0 & Unstable & -1 & 0 & Arbitrary \\
$e_{1}$ & 1 & 0 & Semi-hyperbolic & 1 & 0 & Arbitrary \\
$e_{2}$ & -1 & 0 & Semi-hyperbolic & 1 & 0 & Arbitrary \\
\hline
\end{tabular}

Therefore the equilibrium points and their characteristics on this invariant plane can be summarized in Table 1 .

\subsection{The invariant plane $s=0$}

On this plane system (7) becomes

$\frac{d x}{d t}=x\left(3 x^{2}-2 z^{2}-3\right), \frac{d z}{d t}=z\left(3 x^{2}-2 z^{2}-2\right)$,

which has three equilibrium points $e_{0}=(0,0), e_{1}=(1,0)$ and $e_{2}=(-1,0)$. Here $e_{0}$ is a hyperbolic stable node with eigenvalues -3 and -2 , both $e_{1}$ and $e_{2}$ are unstable hyperbolic nodes with eigenvalues 6 and 1 .

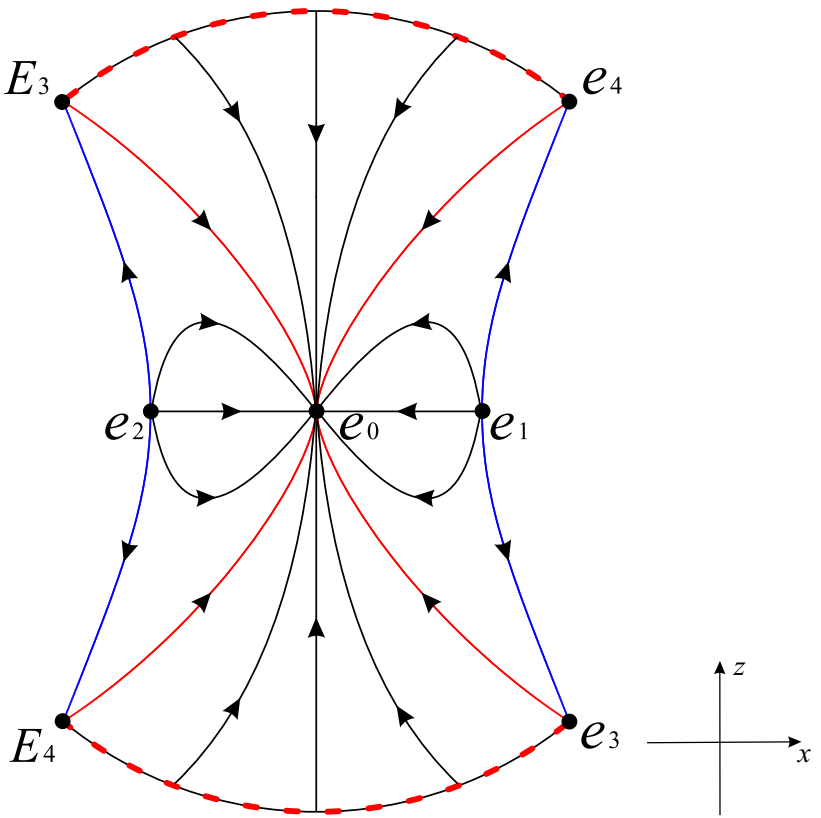

Fig. 2 The phase portrait on the invariant plane $s=0$ restricted to the region $x^{2}-z^{2} \leq 1$

On the local chart $U_{1}$ (see Chapter 5 of [24] for more details on the Poincaré compactification) system (9) becomes

$\frac{d u}{d t}=u v^{2}, \frac{d v}{d t}=v\left(-3+2 u^{2}+3 v^{2}\right)$.

Since this system vanishes at $v=0$, all the points at infinity are equilibrium points. Taking the transformation with respect to time $d \tau=v d t$ yields

$\frac{d u}{d \tau}=u v, \frac{d v}{d \tau}=-3+2 u^{2}+3 v^{2}$.

This system has two hyperbolic points at infinity, $e_{3}=$ $(-\sqrt{6} / 2,0)$ and $e_{4}=(\sqrt{6} / 2,0)$, both of them are unstable hyperbolic saddle points with eigenvalues $\pm \sqrt{6}$.

On the local chart $U_{2}$ system (9) writes

$\frac{d u}{d t}=-u v^{2}, \frac{d v}{d t}=v\left(2-3 u^{2}+2 v^{2}\right)$.

Rescaling the time of system (12) by letting $d \tau=v d t$ we obtain

$\frac{d u}{d \tau}=-u v, \frac{d v}{d \tau}=2-3 u^{2}+2 v^{2}$.

In view of $(0,0)$ is not an equilibrium point of system (13), we will not continue to study other infinite equilibrium points of system (13) because they have been studied in local chart $U_{1}$.

Therefore the global phase portraits of system (9) can be found in Fig. 2. In addition, similar to the previous section, the equilibrium points on this invariant plane and their characteristics are shown in Table 2. 
Table 2 Equilibrium points and their characteristics on the invariant plane $s=0$

\begin{tabular}{lllllll}
\hline $\begin{array}{l}\text { Equilibrium } \\
\text { points }\end{array}$ & $x$ & $z$ & Stability & $\omega_{M}$ & $\Omega_{D E}$ & $\omega_{D E}$ \\
\hline$e_{0}$ & 0 & 0 & Stable & -1 & 0 & Arbitrary \\
$e_{1}$ & 1 & 0 & Unstable & 1 & 0 & Arbitrary \\
$e_{2}$ & -1 & 0 & Unstable & 1 & 0 & Arbitrary \\
$e_{3}$ & $\infty$ & $\infty$ & Unstable & - & $-\infty$ & Arbitrary \\
$e_{4}$ & $\infty$ & $\infty$ & Unstable & - & $-\infty$ & Arbitrary \\
\hline
\end{tabular}

\subsection{The invariant surface $x^{2}-z^{2}=1$}

First we prove that the surface $x^{2}-z^{2}=1$ is invariant under the flow of system (7). If $l=l(x, z, s)=x^{2}-z^{2}-1$, then in order that the surface $x^{2}-z^{2}=1$ be invariant we must have

$\frac{\partial l}{\partial x} \dot{x}+\frac{\partial l}{\partial z} \dot{z}+\frac{\partial l}{\partial s} \dot{s}=K l$,

for some polynomial $K$, and this is the case with $K=$ $2\left(3 x^{2}-2 z^{2}-\sqrt{6} x s\right)$.

On the surface $x^{2}-z^{2}=1$ system (7) can be written as

$\frac{d x}{d t}=x\left(x^{2}-1\right), \frac{d s}{d t}=\frac{\sqrt{6}}{n} x s^{2}$.

Then except for all the points on $x=0$ which are equilibrium points, system (14) also admits two finite equilibrium points $e_{1}=(1,0)$ and $e_{2}=(-1,0)$. By using Theorem 2.19 of [24], we can find that both $e_{1}$ and $e_{2}$ are semi-hyperbolic saddle-nodes.

On the local chart $U_{1}$ system (14) becomes

$\frac{d u}{d t}=u\left(\frac{\sqrt{6}}{n} u+v^{2}-1\right), \frac{d v}{d t}=v\left(v^{2}-1\right)$.

It has two infinite equilibrium points $e_{5}=(0,0)$ and $e_{6}=$ $(\sqrt{6} n / 6,0)$, where $e_{5}$ is a hyperbolic stable node with eigenvalues -1 of multiplicity two, and $e_{6}$ is a hyperbolic unstable saddle point with eigenvalues \pm 1 .

On the local chart $U_{2}$ system (14) writes

$\frac{d u}{d t}=u\left(-\frac{\sqrt{6}}{n} u+u^{2}-v^{2}\right), \frac{d v}{d t}=-\frac{\sqrt{6}}{n} u v$.

Let $d \tau=u d t$ we obtain

$\frac{d u}{d \tau}=-\frac{\sqrt{6}}{n} u+u^{2}-v^{2}, \frac{d v}{d \tau}=-\frac{\sqrt{6}}{n} v$.

The origin $e_{7}=(0,0)$ on the local chart $U_{2}$ is a hyperbolic stable node with eigenvalues $-\sqrt{6} / n$ of multiplicity two.

In short the global phase portraits of system (14) is shown in Fig. 3. Furthermore, similar to the previous Sects. 3.1 and

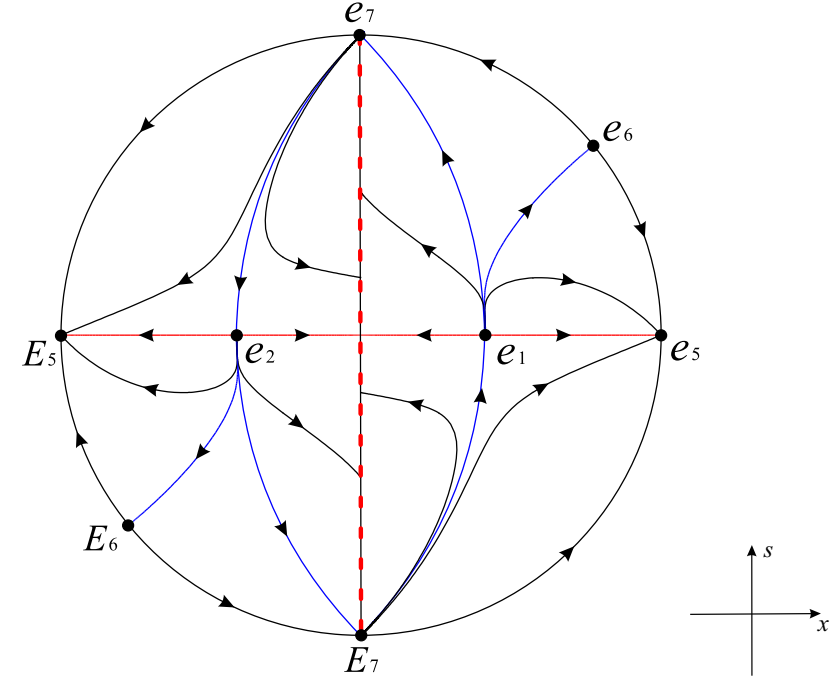

Fig. 3 The phase portrait of the invariant surface $x^{2}-z^{2}=1$

Table 3 Equilibrium points and their characteristics on the invariant plane $x^{2}-z^{2}=1$ for all $s \in \mathbb{R}$

\begin{tabular}{lllllll}
\hline $\begin{array}{l}\text { Equilibrium } \\
\text { points }\end{array}$ & $x$ & $z$ & Stability & $\omega_{M}$ & $\Omega_{D E}$ & $\omega_{D E}$ \\
\hline$e_{1}$ & 1 & 0 & Semi-hyperbolic & 1 & 0 & Arbitrary \\
$e_{2}$ & -1 & 0 & Semi-hyperbolic & 1 & 0 & Arbitrary \\
$e_{5}$ & $\infty$ & $\infty$ & Stable & 1 & $-\infty$ & Arbitrary \\
$e_{6}$ & $\infty$ & $\infty$ & Unstable & 1 & $-\infty$ & Arbitrary \\
\hline
\end{tabular}

3.2, the equilibrium points and their characteristics on this invariant surface are collated in Table 3.

\subsection{The finite equilibrium points}

It is noted that system (7) admits three finite equilibrium points $p_{0}=(0,0,0)$ with eigenvalues $\{-3,-2,0\}, p_{1}=$ $(1,0,0)$ and $p_{2}=(-1,0,0)$ with the same eigenvalues $\{6,1,0\}$. Here $p_{1}$ and $p_{2}$ are located at the intersection of the invariant planes $z=0, s=0$ and the invariant surface $x^{2}-z^{2}=1$, corresponding to the equilibrium points $e_{1}$ and $e_{2}$ in Sects. 3.1-3.3, respectively. The origin $p_{0}$ of system (7) lies at the intersection of the invariant planes $z=0$ and $s=0$, which is the same point as the equilibrium point $e_{0}$ studied in Sects. 3.1 and 3.2.

\section{Phase portraits on the Poincaré sphere at infinity}

In order to describe the dynamics of system (7) at infinity. We use the method of the three-dimensional Poincaré compactification (see [25] for more details) $x=1 / z_{3}, z=z_{1} / z_{3}, s=$ $z_{2} / z_{3}$, then the analytical vector field of system (7) on the 
Table 4 Equilibrium points on the different local charts of the Poincaré sphere at the infinity of $\mathbb{R}^{3}$

\begin{tabular}{ll}
\hline Equilibrium points & Eigenvalues \\
\hline$u_{11}=(0,0,0)$ & $\{-3,-3,0\}$ \\
$u_{12}=\left(-\frac{\sqrt{6}}{2}, 0,0\right)$ & $\{0,0,0\}$ \\
$u_{13}=\left(\frac{\sqrt{6}}{2}, 0,0\right)$ & $\{0,0,0\}$ \\
$u_{14}=\left(-1, \frac{\sqrt{6}}{6} n, 0\right)$ & $\{-1,1,-2 n\}$ \\
$u_{15}=\left(1, \frac{\sqrt{6}}{6} n, 0\right)$ & $\{-1,1,-2 n\}$ \\
$u_{16}=\left(0, \frac{\sqrt{6}}{2} \frac{n}{1+n}, 0\right)$ & $\left\{-\frac{3}{1+n}, \frac{3 n}{1+n}, 3\right\}$ \\
$u_{b 0}=(b, 0,0)$ & $\left\{0,-3+2 b^{2},-3+2 b^{2}\right\}$ \\
$u_{31}=(0,0,0)$ & $\{0,0,0\}$ \\
\hline
\end{tabular}

local chart $U_{1}$ becomes

$\frac{d z_{1}}{d t}=z_{1}\left[z_{3}^{2}-\sqrt{6} z_{2}\left(-1+z_{1}^{2}+z_{3}^{2}\right)\right]$,

$\frac{d z_{2}}{d t}=z_{2}\left[-3+2 z_{1}^{2}+3 z_{3}^{2}+\sqrt{6} z_{2}\left(\frac{1}{n}+1-z_{1}^{2}-z_{3}^{2}\right)\right]$,

$\frac{d z_{3}}{d t}=-z_{3}\left[z_{1}^{2}\left(-2+\sqrt{6} z_{2}\right)+\left(-3+\sqrt{6} z_{2}\right)\left(-1+z_{3}^{2}\right)\right]$.

In the different local charts of the Poincare sphere, the infinity of $\mathbb{R}^{3}$ corresponds to $z_{3}=0$, then system (18) has the equilibrium points listed in Table 4 . Except that the equilibrium point $u_{31}$ denotes the origin of the local chart $U_{3}$, the rest equilibrium points lie in the local chart $U_{1}$. Moreover the straight line $s=0$ of the local chart $U_{1}$ is filled with the equilibrium points $u_{b 0}$ for all $b \in \mathbb{R}$.

For the case $z_{3}=0$ system (18) is reduced to

$\frac{d z_{1}}{d t}=-\sqrt{6} z_{1} z_{2}\left(-1+z_{1}^{2}\right)$

$\frac{d z_{2}}{d t}=z_{2}\left[-3+2 z_{1}^{2}+\sqrt{6} z_{2}\left(\frac{1}{n}+1-z_{1}^{2}\right)\right]$.

After changing the of time $d \tau=z_{2} d t$ system (19) becomes

$\frac{d z_{1}}{d \tau}=-\sqrt{6} z_{1}\left(-1+z_{1}^{2}\right)$,

$\frac{d z_{2}}{d \tau}=-3+2 z_{1}^{2}+\sqrt{6} z_{2}\left(\frac{1}{n}+1-z_{1}^{2}\right)$.

Then this system has three equilibrium points $e_{i, 1}, e_{i, 2}$ and $e_{i, 3}$ with coordinates $(-1, \sqrt{6} n / 6),(1, \sqrt{6} n / 6)$ and $(0, \sqrt{6} n /(2(1+n)))$, respectively. Here both $e_{i, 1}$ and $e_{i, 2}$ are unstable saddle points with eigenvalues $\sqrt{6} / n$ and $-2 \sqrt{6}$. $e_{i, 3}$ is an unstable node with eigenvalues $\sqrt{6}$ and $\sqrt{6}(1+$

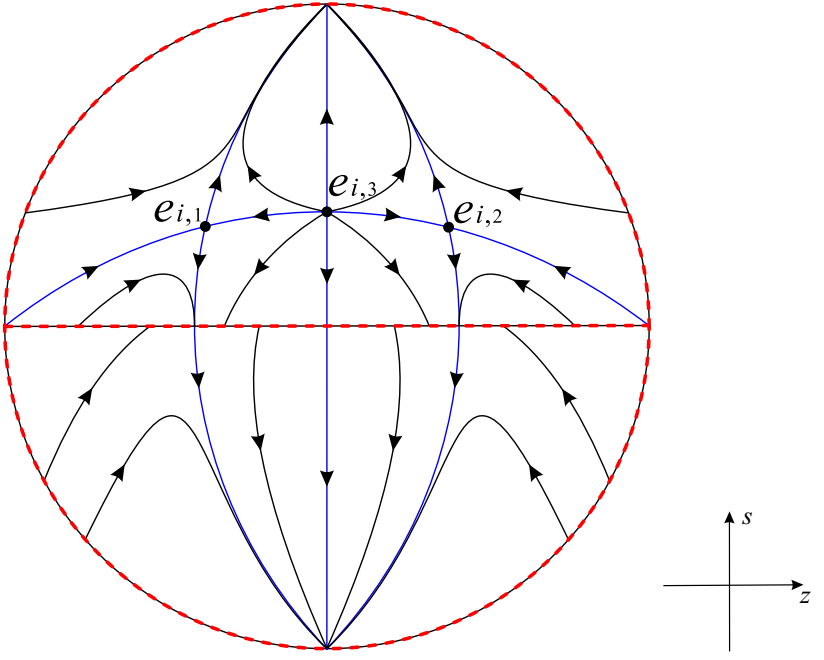

Fig. 4 The phase portrait of system (7) at infinity on local chart $U_{1}$

$n) / n$. The phase portrait on the Poincaré sphere at infinity on local chart $U_{1}$ is shown in Fig. 4.

On the local chart $U_{2}$ in view of Poincaré compactification $x=z_{1} / z_{3}, z=1 / z_{3}, s=z_{2} / z_{3}$, system (7) writes

$\frac{d z_{1}}{d t}=-z_{1} z_{3}^{2}+\sqrt{6} z_{2}\left(1-z_{1}^{2}+z_{3}^{2}\right)$,

$\frac{d z_{2}}{d t}=z_{2}\left(2-3 z_{1}^{2}+\frac{\sqrt{6}}{n} z_{1} z_{2}+2 z_{3}^{2}\right)$,

$\frac{d z_{3}}{d t}=z_{3}\left(2-3 z_{1}^{2}+2 z_{3}^{2}\right)$.

Since we want to study the infinity we take $z_{3}=0$, rescaling the time $d \tau=z_{2} d t$ system (21) is equivalent to

$$
\begin{aligned}
& \frac{d z_{1}}{d \tau}=\sqrt{6}\left(1-z_{1}^{2}\right), \\
& \frac{d z_{2}}{d \tau}=2-3 z_{1}^{2}+\frac{\sqrt{6}}{n} z_{1} z_{2} .
\end{aligned}
$$

For any constant $c$, since $(c, 0)$ is not the equilibrium point of the system (22), we will not continue to discuss other infinite equilibrium points of the system (22). Because this has been discussed in the case $U_{1}$.

On the local chart $U_{3}$ the three-dimensional Poincaré compactification is $x=z_{1} / z_{3}, z=z_{2} / z_{3}, s=1 / z_{3}$, then system (7) becomes

$$
\begin{aligned}
\frac{d z_{1}}{d t}= & z_{1}\left[-\frac{\sqrt{6}(1+n)}{n} z_{1}+3 z_{1}^{2}-2 z_{2}^{2}-3 z_{3}^{2}\right] \\
& +\sqrt{6}\left(z_{2}^{2}+z_{3}^{2}\right), \\
\frac{d z_{2}}{d t}= & z_{2}\left[-\frac{\sqrt{6}}{n} z_{1}+3 z_{1}^{2}-2\left(z_{2}^{2}+z_{3}^{2}\right)\right], \\
\frac{d z_{3}}{d t}= & -\frac{\sqrt{6}}{n} z_{1} z_{3} .
\end{aligned}
$$


For the case $z_{3}=0$ from system (23) we obtain

$$
\begin{aligned}
& \frac{d z_{1}}{d t}=z_{1}\left[-\frac{\sqrt{6}(1+n)}{n} z_{1}+3 z_{1}^{2}-2 z_{2}^{2}\right]+\sqrt{6} z_{2}^{2}, \\
& \frac{d z_{2}}{d t}=z_{2}\left(-\frac{\sqrt{6}}{n} z_{1}+3 z_{1}^{2}-2 z_{2}^{2}\right) .
\end{aligned}
$$

It is noted that $(0,0)$ is a linearly zero equilibrium point, the topological index is zero from the Poincaré-Hopf theory (see Theorem 6.30 of [24] for more details). In order to study its local phase portrait we shall use vertical blow-ups (see for instance [26]) by letting $w=z_{2} / z_{1}$, then we obtain

$$
\begin{aligned}
& \frac{d z_{1}}{d t}=z_{1}^{2}\left[\sqrt{6}\left(-\frac{1}{n}-1+w^{2}\right)+\left(3-2 w^{2}\right) z_{1}\right], \\
& \frac{d w}{d t}=-\sqrt{6} z_{1} w\left(-1+w^{2}\right) .
\end{aligned}
$$

Eliminating the common factor $z_{1}$ of system (25) by changing the time $d \tau=z_{1} d t$ it yields

$$
\begin{aligned}
& \frac{d z_{1}}{d \tau}=z_{1}\left[\sqrt{6}\left(-\frac{1}{n}-1+w^{2}\right)+\left(3-2 w^{2}\right) z_{1}\right], \\
& \frac{d w}{d \tau}=-\sqrt{6} w\left(-1+w^{2}\right) .
\end{aligned}
$$

System (26) admits three equilibrium points $e_{i, 4}=(0,-1)$, $e_{i, 5}=(0,1)$ and $e_{i, 6}=(0,0)$ on $z_{1}=0$, where $e_{i, 4}$ and $e_{i, 5}$ are two hyperbolic stable nodes with eigenvalues $-\sqrt{6} / n$ and $-2 \sqrt{6}, e_{i, 6}$ is a hyperbolic unstable saddle point with eigenvalues $\sqrt{6}$ and $-\sqrt{6}(1+n) / n$. The local phase portraits around $e_{i, 4}, e_{i, 5}$ and $e_{i, 6}$ are shown in Fig. 5a. Note that there is a time rescaling $d \tau=z_{1} d t$ between systems (25) and (26), so the direction of the trajectories in the local phase portraits of system (25) is opposite to that of Fig. 5a when $z_{1}<0$, see Fig. 5b for more details. In addition, all points on the $w$ axis, i.e., $z_{1}=0$, are singularities of system (25). Thus the local phase portraits at $(0,0)$ of system $(24)$ is shown in Fig. 5c. Then the phase portrait in the local chart $U_{3}$ is shown in Fig. 6.

In summary joining the previous information we obtain the global phase portraits at infinity in the Poincaré sphere in Fig. 7 .

\section{Phase portraits inside the Poincaré ball restricted to $x^{2}-z^{2} \leq 1$}

It is noted that system (7) is invariant under the symmetries $(x, z, s) \mapsto(-x,-z,-s)$ and $(x, z, s) \mapsto(-x, z,-s)$, so it is invariant under the symmetry with respect to the origin and to the $z$-axis. Now we divide the Poincaré ball restricted to $x^{2}-z^{2} \leq 1$ into the following four regions:

$R_{1}: z \leq 0, s \geq 0 . \quad R_{2}: z \leq 0, s \leq 0$.

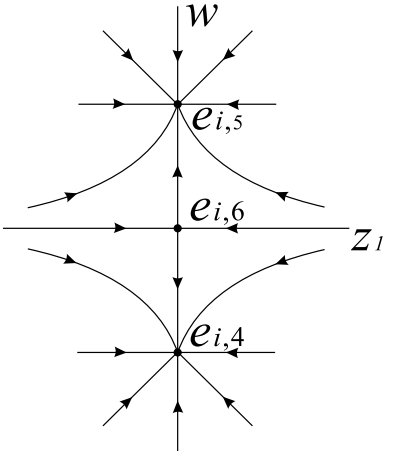

(a)

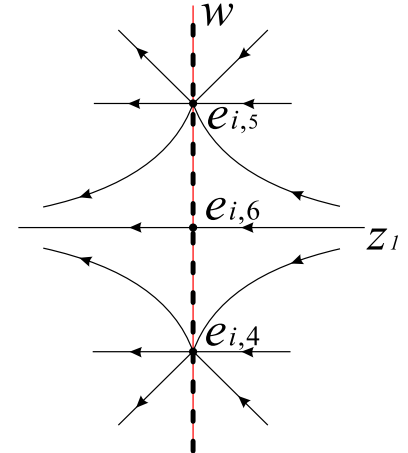

(b)

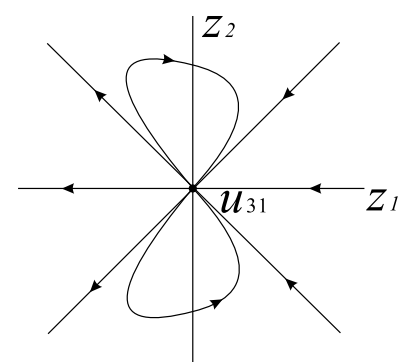

(c)

Fig. 5 In a-c there are the local phase portraits of the equilibrium points in systems (26), (25) and (24), respectively

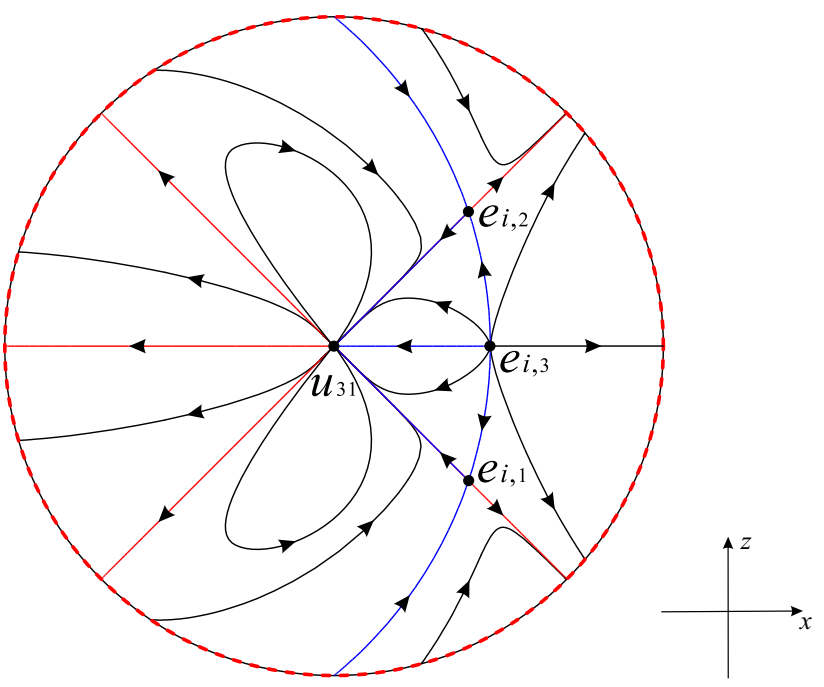

Fig. 6 The phase portrait of system (24), i.e., the phase portrait at infinity in the local chart $U_{3}$ of system (7)

$R_{3}: z \geq 0, s \geq 0 . \quad R_{4}: z \geq 0, s \leq 0$.

Then due to the symmetries we only need to study the phase portraits of system (7) in the region $R_{1}$.

Combining the phase portraits of the invariant surface $x^{2}-$ $z^{2}=1$ with the phase portraits of the planes $z=0$, and $s=0$, together with the phase portrait at infinity, we get the phase portraits on the boundary of the region $R_{1}$ as shown in Figs. 8, 9 and 10. Here we explain the definition of the 


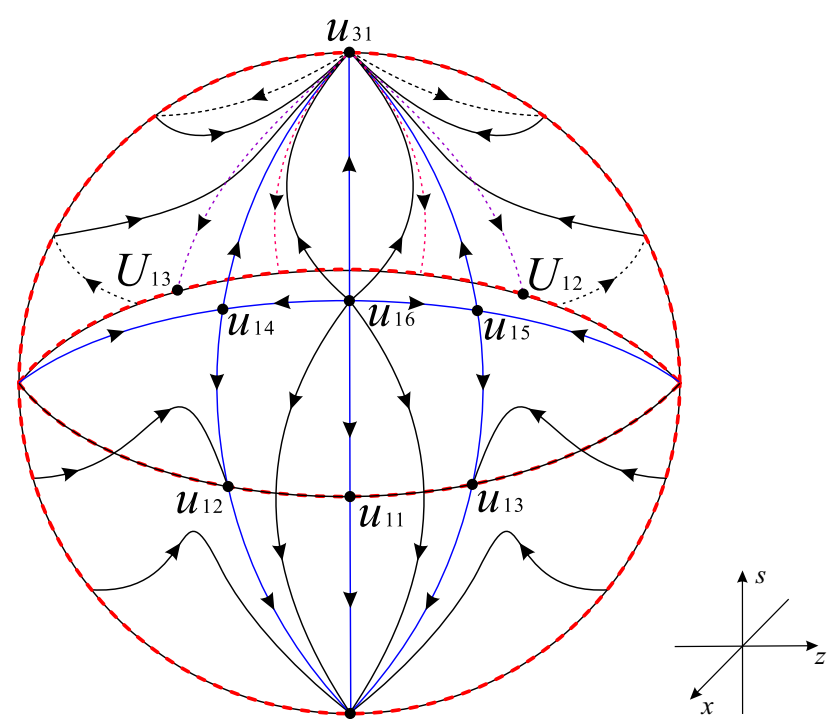

Fig. 7 The global phase portrait at infinity in the Poincaré sphere. The North Pole of the Poincaré ball is the equilibrium point $u_{31}$. The symmetric points of $u_{12}$ and $u_{13}$ with respect to the center of the sphere are $U_{12}$ and $U_{13}$, respectively
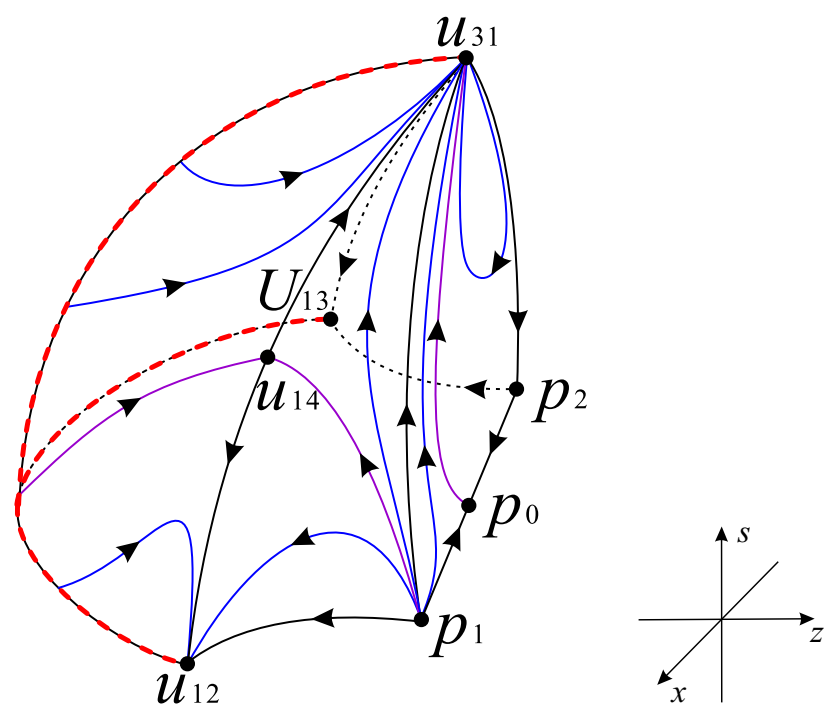

Fig. 8 Phase portrait in the front boundary of the region $R_{1}$

three-dimensional cartesian coordinate system in this paper as follows: we regard the $x z$-plane as the horizontal plane in $\mathbb{R}^{3}$, where the direction of the $z$-axis is horizontal towards the right. If the $z$-axis axis rotates $90^{\circ}$ clockwise we get the $x$ axis. The $s$-axis is vertical upward, and then $x z s$ constitutes a three-dimensional right-handed cartesian coordinate system.

In order to show the phase portraits more clearly, the boundary of the region $R_{1}$ is divided into six surfaces according to the orientation towards us, the back to us and the bottom area. See Fig. 11 for more details. It is noted that the North Pole $u_{31}$ of the Poincaré ball on the front boundary surfaces $F_{1}$ and $F_{2}$ is stable, and there is an elliptic sector and a stable
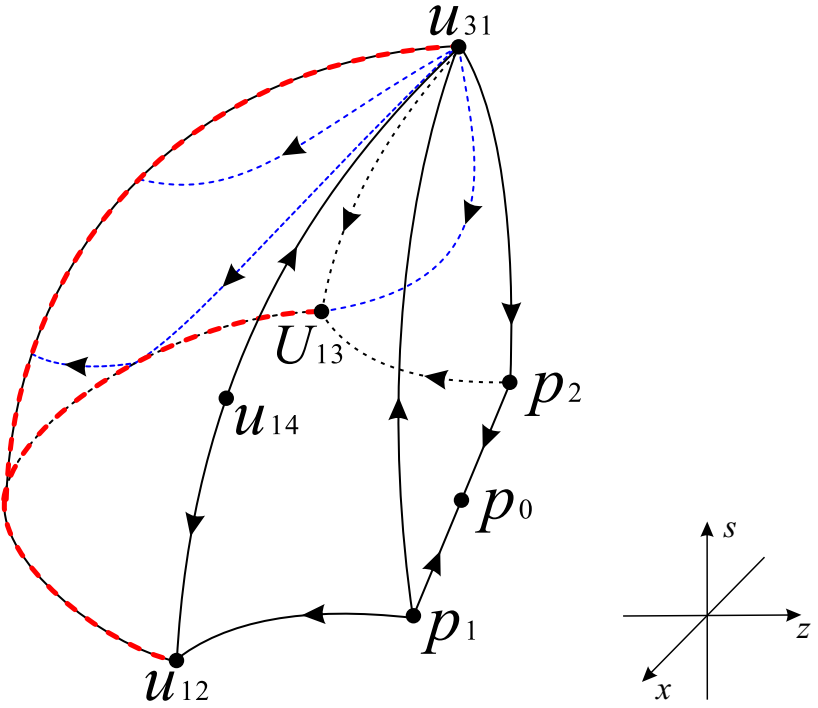

Fig. 9 Phase portrait in the back boundary of the region $R_{1}$
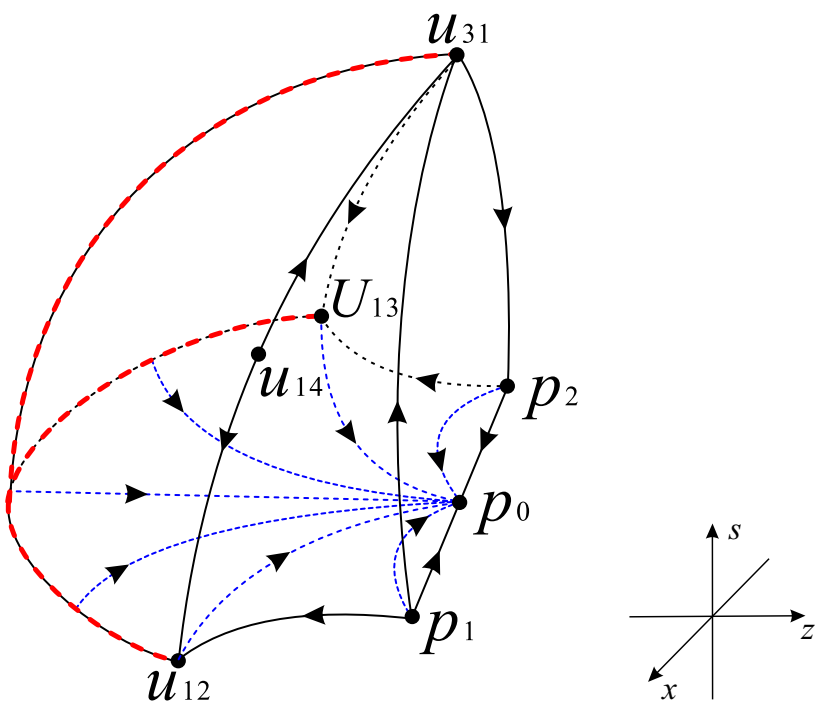

Fig. 10 Phase portrait in the bottom of the region $R_{1}$

parabolic sector segment of $U_{3}$ on the boundary $F_{3}$, but it is unstable on the back boundary surfaces $B_{1}$ and $B_{2}$.

\section{Dynamics in the interior of the region $R_{1}$}

Note that the original system (7) admits the three finite equilibrium points $p_{0}, p_{1}$ and $p_{2}$ in the three-dimensional cartesian coordinate system. The dynamical behavior of the system inside the region $R_{1}$ depends on the behavior of the flow in the following surfaces and planes

$h(x, z, s)=0, g(x, z)=0, x=0, z=0, s=0$, 
Fig. 11 The six boundary surfaces of the region $R_{1}$
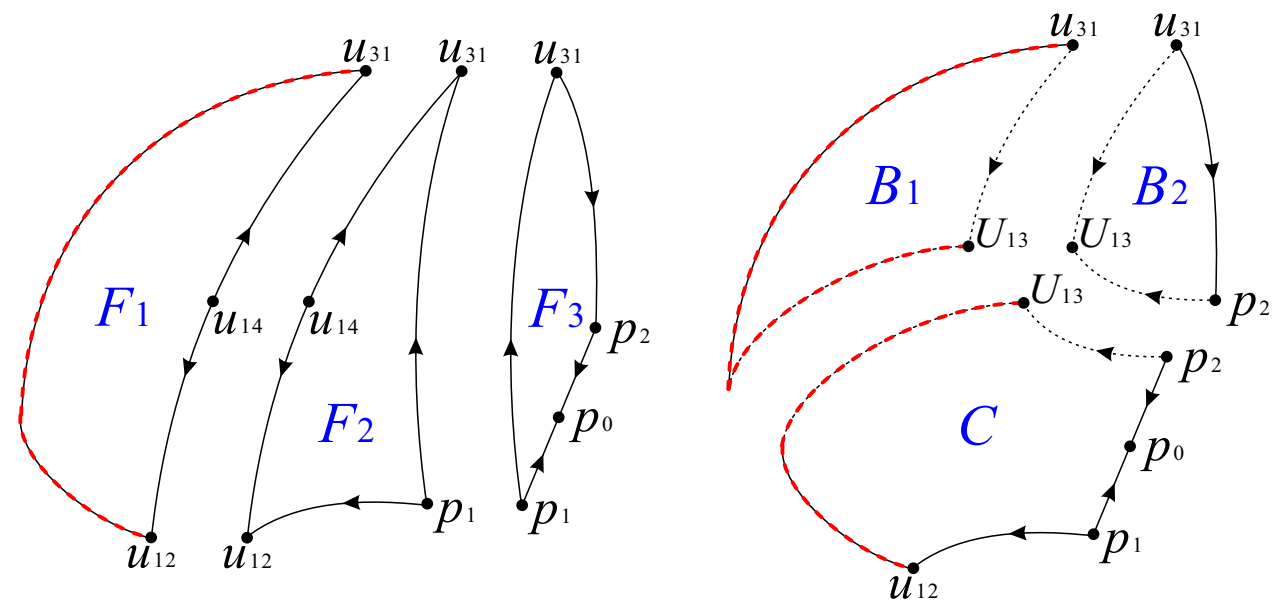
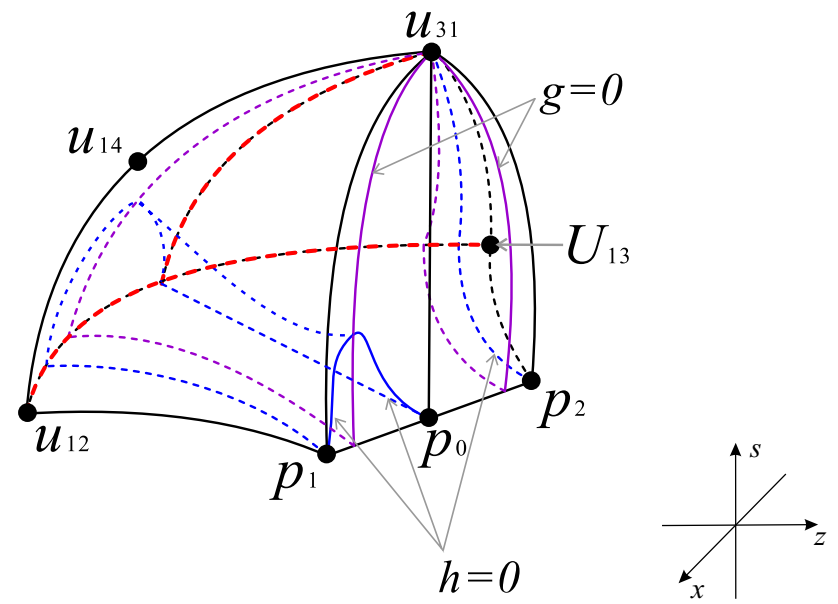

Fig. 12 There are seven subregions inside the region $R_{1}$ of the Poincaré ball

where

$$
\begin{aligned}
h(x, z, s) & =x\left(3 x^{2}-2 z^{2}-3\right)+\sqrt{6} s\left(1-x^{2}+z^{2}\right), \\
g(x, z) & =3 x^{2}-2 z^{2}-2 .
\end{aligned}
$$

These surfaces and planes divide the region $R_{1}$ into seven different subregions $R_{1 i}, i=(1,2, \ldots, 7)$, see Figs. 12, $13,14,15,16,17,18$ and 19 for more details. It should be noted that $R_{11}$ and $R_{13}$ represent parallel tunnels inside $R_{1}$, respectively. It is easy to verify that $h>0$ in the subregions $R_{12}, R_{14}, R_{15}$ and $R_{16}$, and $h<0$ in the subregions $R_{11}, R_{13}$ and $R_{17}$. Similarly, we can find that $g>0$ in the subregions $R_{11}, R_{12}, R_{16}$ and $R_{17}$, and $g<0$ in the subregions $R_{13}, R_{14}$ and $R_{15}$. It should be noted that the dotted and solid lines in Figs. 13, 14, 15, 16, 17, 18 and 19 are consistent with those in Fig. 12.

As shown in the subregion $R_{11}$ (see Fig. 13) the front surface consists of three dashed lines and one solid line contained in the surface $h=0$, and the surface on the back side

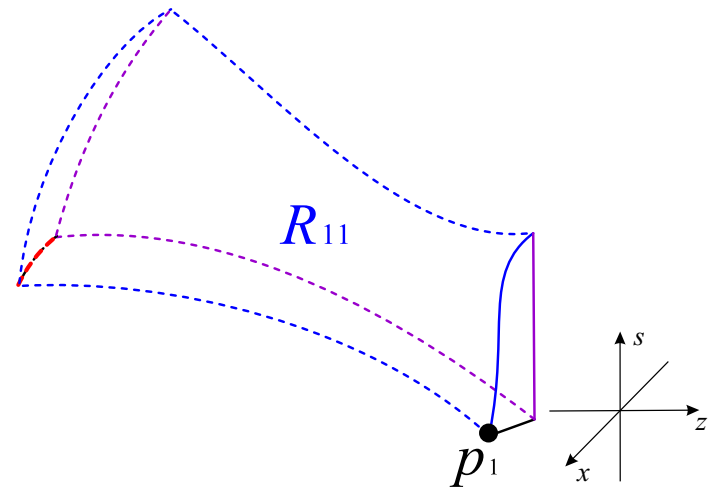

Fig. 13 The subregion $R_{11}$
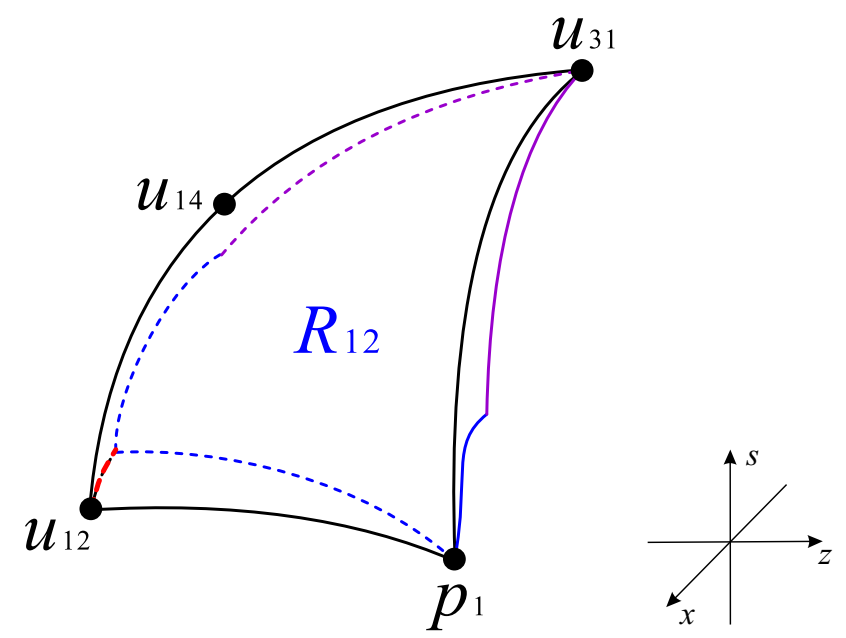

Fig. 14 The subregion $R_{12}$

(opposite to the above front surface) contained in the surface $g=0$.

In Table 5 we describe the behavior of $\dot{x}, \dot{z}$ and $\dot{s}$ in the seven subregions $R_{11}, \ldots, R_{17}$. From this table we obtain that the variables $x$ and $z$ in the subregion $R_{11}$ decrease monotonically, and the variable $s$ increases monotonically, so an orbit in the subregion $R_{11}$ either crosses the boundary $h=0$ 


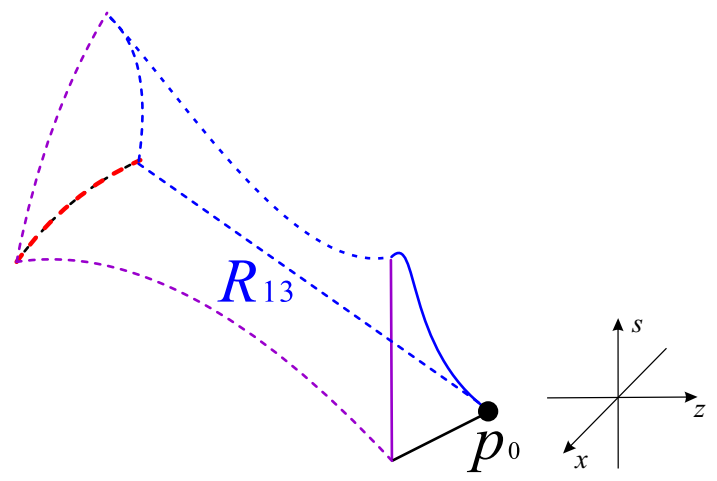

Fig. 15 The subregion $R_{13}$
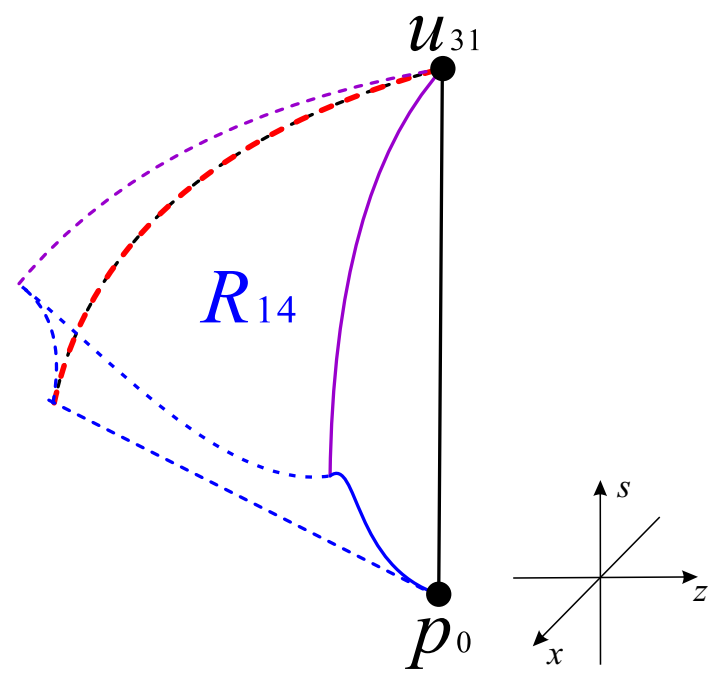

Fig. 16 The subregion $R_{14}$
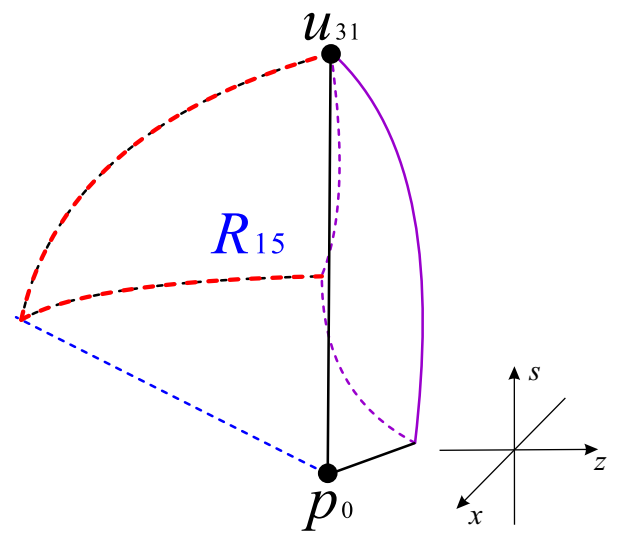

Fig. 17 The subregion $R_{15}$

and enters into the subregion $R_{12}$, or crosses the boundary $g=0$ and goes to the subregion $R_{13}$, or crosses the intersection curve of the subregions $R_{11}, R_{12}$ and $R_{13}$ into the subregion $R_{14}$ with very low probability, i.e. an orbit in the subregion $R_{11}$ will not stay in the future in this region, but will exit through its boundaries into other subregions, and

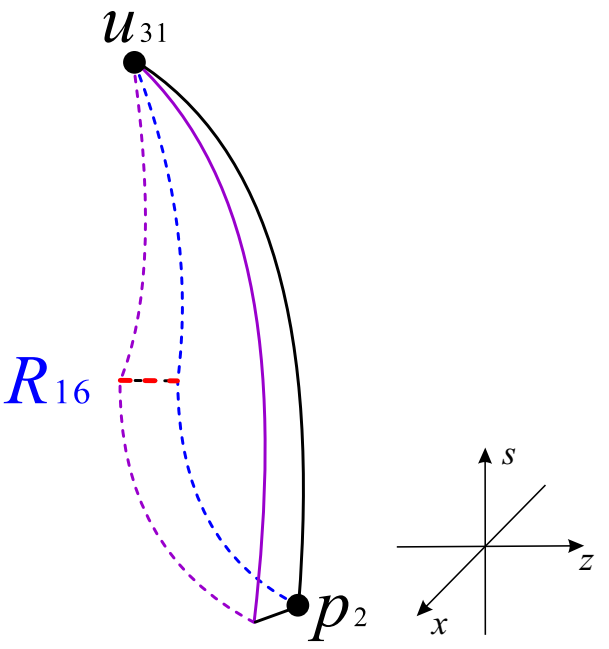

Fig. 18 The subregion $R_{16}$
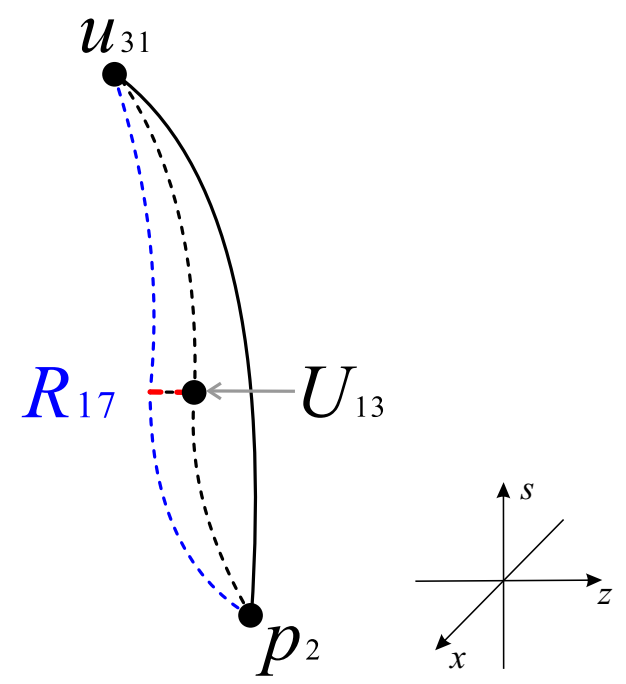

Fig. 19 The subregion $R_{17}$

these orbits in backwards time come from the equilibrium point $p_{1}$ in the subregion $R_{11}$. This process can be simply summarized as follows

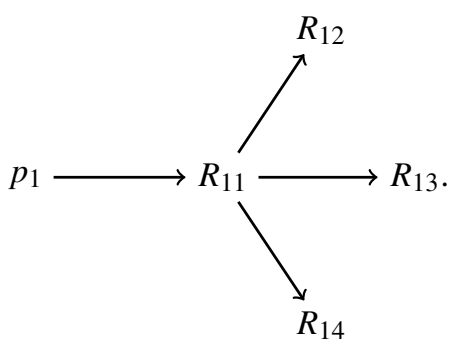

The front boundary of $R_{12}$ (see Fig. 14), i.e. the surface containing the equilibrium points $u_{12}, u_{14}, u_{31}$ and $p_{1}$, is contained in the invariant surface $x^{2}-z^{2}=1(x>0)$. The surface on the back side of $R_{12}$ that contains the equilibrium points $p_{1}$ and $u_{31}$ consists of two parts: the upper part surface 
Table 5 Dynamical behavior in seven different subregions

\begin{tabular}{lll}
\hline Subregions & Corresponding region & Increase or decrease \\
\hline$R_{11}$ & $h<0, g>0, x>0, z<0, s>0$ & $\dot{x}<0, \dot{z}<0, \dot{s}>0$ \\
$R_{12}$ & $h>0, g>0, x>0, z<0, s>0$ & $\dot{x}>0, \dot{z}<0, \dot{s}>0$ \\
$R_{13}$ & $h<0, g<0, x>0, z<0, s>0$ & $\dot{x}<0, \dot{z}>0, \dot{s}>0$ \\
$R_{14}$ & $h>0, g<0, x>0, z<0, s>0$ & $\dot{x}>0, \dot{z}>0, \dot{s}>0$ \\
$R_{15}$ & $h>0, g<0, x>0, z<0, s>0$ & $\dot{x}>0, \dot{z}>0, \dot{s}<0$ \\
$R_{16}$ & $h>0, g>0, x>0, z<0, s>0$ & $\dot{x}>0, \dot{z}<0, \dot{s}<0$ \\
$R_{17}$ & $h<0, g>0, x>0, z<0, s>0$ & $\dot{x}<0, \dot{z}<0, \dot{s}<0$ \\
\hline
\end{tabular}

containing the infinite equilibrium point $u_{31}$ is the intersection of the subregions $R_{12}$ and $R_{14}$, which is contained in the surface $g=0$, and the lower part surface containing the finite equilibrium point $p_{1}$, is the intersection of subregions $R_{11}$ and $R_{12}$ that is contained in the surface $h=0$. The equilibrium points in the subregion $R_{12}$ are the finite equilibrium point $p_{1}$, the infinite equilibrium points on the Poincaré sphere at $s=0$ and the equilibrium point $u_{14}$ at infinity. However an orbit in the subregion $R_{12}$ does not start from these infinite equilibrium points at $s=0$ or return to these points, but it comes from the finite equilibrium point $p_{1}$ or from the subregions $R_{11}$ and $R_{14}$ which have common boundaries with the subregion $R_{12}$. This is due to the fact that the variable $z$ is decreasing monotonically and the variables $x$ and $s$ are increasing monotonically inside the subregion $R_{12}$ according to Table 5. Moreover a trajectory in the subregion $R_{12}$ will tend to the equilibrium point $u_{14}$ at infinity. In summary, this dynamic behavior process can be represented as

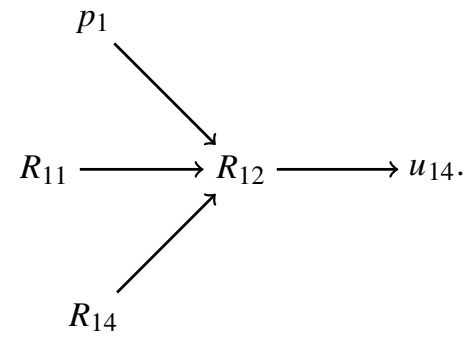

In the subregion $R_{13}$ (see Fig. 15) the front surface composed of three dashed lines and one solid line is contained in the surface $g=0$, and its opposite surface is contained in the surface $h=0$. From Table 5 an orbit in the subregion $R_{13}$ may only comes from the equilibrium points locate at the infinity on $s=0$ in $R_{13}$, or it comes from the subregions $R_{11}$. Then the orbit passes through the intersection of subregions $R_{13}$ and $R_{14}$ (contained in the surface $h=0$ ) and enters the subregion $R_{14}$, or tends to the infinite equilibrium point $u_{31}$, i.e., lies in the North Pole of the Poincaré sphere. So we obtain the following dynamics

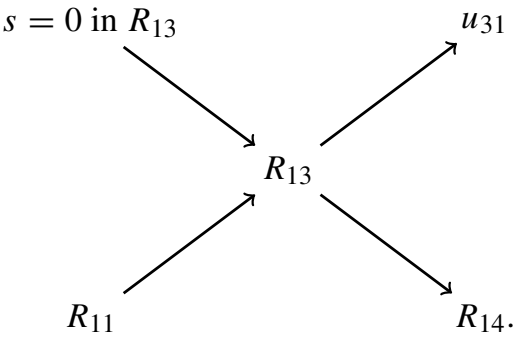

In subregion $R_{14}$ (see Fig. 16) the front triangular surface is contained in $g=0$, and its opposite triangular surface is on the back side, which is included in the plane $x=0$. According to Table 5 an orbit of the subregion $R_{14}$ may come from the infinite equilibrium points on $x=0$, or come from the subregion $R_{13}$ through the surface $h=0$, or from subregion $R_{15}$ traversing plane $x=0$, then go through the surface $g=0$ into the subregion $R_{12}$, or directly goes to the infinite equilibrium point $u_{31}$ at the North Pole of the Poincaré sphere. This can be represented as

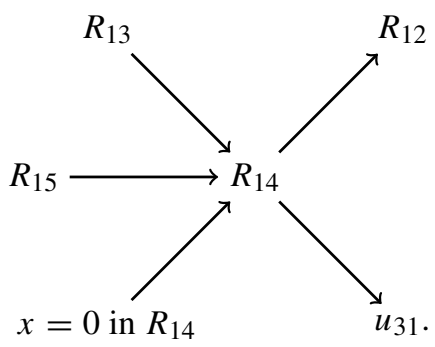

It can be noted from Fig. 17 that the surface on the left of subregion $R_{15}$ (consisting of two dashed lines and one solid line) is contained in plane $x=0$, and the opposite surface to it is included in the surface $g=0$. According to Table 5 an orbit in the subregion $R_{15}$ can only come from the adjacent subregion $R_{16}$, and then either enter into the subregion $R_{14}$ adjacent to it, or directly tend to the finite equilibrium point $p_{0}$ located at the center of the Poincaré ball. This dynamics can be denoted as 


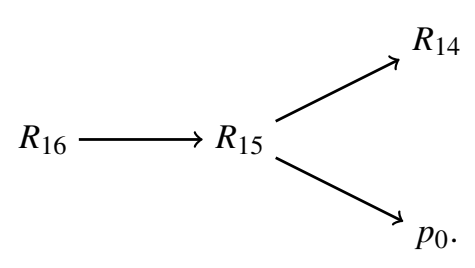

We observe that the curved surfaces of the left and right sides of the subregion $R_{16}$ (see Fig. 18) are contained in the surfaces $g=0$ and $h=0$, respectively. It is known from Table 5 that an orbit in the subregion $R_{16}$ can only come from the subregion $R_{17}$ and crossing the surface $g=0$, it enters into the subregion $R_{15}$. Thus we get that

$$
R_{17} \longrightarrow R_{16} \longrightarrow R_{15}
$$

The left and right surfaces of subregion $R_{17}$ (see Fig. 19) are included in the surface $h=0$ and in the invariant surface $x^{2}-z^{2}=1(x<0)$, respectively. According to Table 5, it is known that all three variables in subregion $R_{17}$ are decreasing monotonically, so an orbit in this subregion must come from the infinite equilibrium point $u_{31}$ located at the North Pole of Poincaré sphere and eventually tends to the infinite equilibrium point $U_{13}$ in the invariant plane $s=0$. Then we have that

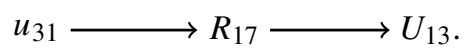

The dynamic behavior of the orbits inside the seven subregions of $R_{1}$ discussed above can be summarized as

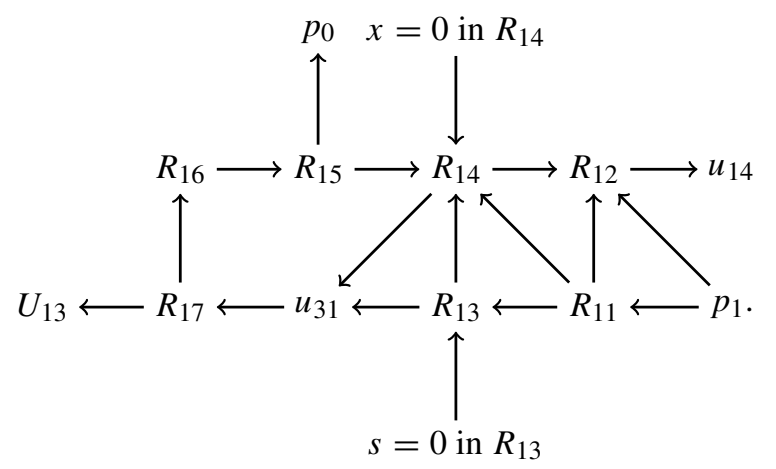

The above flow chart shows that the orbits of system (7) contained in the interior of the region $R_{1}$ have $\alpha$-limit at the finite equilibrium point $p_{1}$, and the some orbits on the boundary of the region $R_{1}$ have $\alpha$-limit at the $x=0$ in $R_{14}$, or $s=0$ in $R_{13}$. Moreover the orbits have $\omega$-limit either at the finite equilibrium point $p_{0}$, or at the infinite equilibrium points $u_{14}$ and $U_{13}$, where $u_{14}$ is located on the intersection curve of the Poincaré sphere and the invariant surface $x^{2}-z^{2}=1(x>0)$ at infinity, and $U_{13}$ is located on the intersection of the Poincaré sphere and the invariant surfaces $s=0, x^{2}-z^{2}=1(x<0)$ at infinity (see Fig. 12). Furthermore the orbits of system (7) on the boundary of $R_{1}$ also have $\alpha$-limit and $\omega$-limit at the infinite equilibrium point $u_{31}$, i.e. the North Pole of the Poincaré sphere.

For the sake of summary, similar to the previous Sects. 3.13.3 , the equilibrium points on the region $R_{1}$ and their characteristics within this region are integrated in Table 6.

In this way the qualitative global dynamic behavior of system (7) is described.

\section{Discussion and conclusions}

From a cosmological point of view, the unstable finite equilibrium points $p_{1}$ and $p_{2}$ as shown in Figs. 8, 9 and 10 or 12 are consistent with the results of case 1 in the cosmological model in $[3,5]$, corresponding to complete dark matter domination. It is known from the last flow chart in Sect. 6 that the physical importance of the finite equilibrium point $p_{0}$ is a late-time state of the universe if the initial condition is not on the invariant plane $z=0$ or on the invariant surface $x^{2}-z^{2}=1$. We also note that this late-time solution was already obtained in the strip $-\sqrt{6} / 2<s<\sqrt{6} / 2$ for the cosmological model with exponential potential in [3-5], but our results hold for all values of $s$ in the region $G$ of the three-dimensional space. In addition, the main differences between the Ref. [5] and the present paper are:

1. The former specifically studied the case of exponential potential, our research is based on a wide range of potentials, and specifically considering the case of power-law potential.

2. The former is based on the planar cosmological model, and we are considering a three-dimensional one.

3. The former did not discussed the dynamics at infinity, but we studied the infinite equilibrium points, and found that the infinite equilibrium points $u_{14}$ and $U_{13}$ may also be the late-time state solutions of the universe.

See the last flow chart in Sect. 6 again, the equilibrium points $u_{14}$ and $U_{13}$ will be future attractors located at infinity. When the initial conditions are inside the region $R_{1}$, they can be the late-time states of the universe. It is noted that only $u_{14}$ and $U_{13}$ are studied in the region $R_{1}$ because system (7) admits two aforementioned symmetries. Thus the results of $u_{15}$ and $u_{13}$ (see Fig. 7) are identical with those of $u_{14}$ and $U_{13}$, respectively. It should also be noted that Fig. 5.5 in [3] or Fig. 3 in [5] can be obtained in Fig. 7 in the present paper when $s=0.6$. Then it is easy to find that $u_{14}$ corresponds to $P_{6}$ in the Fig. 5.5 in [3] for the plane case when $s \rightarrow 0$. However the infinite equilibrium point $u_{14}$ can be the latetime state of the universe from the above analysis, while $P_{6}$ corresponds to the universe dominated by dark matter due to the absence of $\Lambda$ and the effect of $k$ will decrease with the increasing of the scale factor $a(t)$. 
Table 6 Equilibrium points and their characteristics in the interior of the region $R_{1}$ for all $s \in \mathbb{R}$

\begin{tabular}{llllllll}
\hline Equilibrium points & $x$ & $z$ & $s$ & Stability & $\omega_{M}$ & $\Omega_{D E}$ & $\omega_{D E}$ \\
\hline$p_{0}$ & 0 & 0 & 0 & Stable & -1 & 0 & Arbitrary \\
$p_{1}$ & 1 & 0 & 0 & Unstable & 1 & 0 & Arbitrary \\
$p_{2}$ & -1 & 0 & 0 & Unstable & 1 & 0 & Arbitrary \\
$u_{12}$ & $\infty$ & $\infty$ & 0 & Unstable & - & $-\infty$ & Arbitrary \\
$U_{13}$ & $\infty$ & $\infty$ & 0 & Stable & - & $-\infty$ & Arbitrary \\
$u_{14}$ & $\infty$ & $\infty$ & $\infty$ & Stable & - & $-\infty$ & Arbitrary \\
$u_{b 0}$ & $\infty$ & 0 & 0 & Unstable & - & 0 & Arbitrary \\
$u_{31}$ & 0 & 0 & $\infty$ & Unstable & -1 & 0 & Arbitrary \\
\hline
\end{tabular}

As a conclusion, based on Hořava-Lifshitz gravity in a Friedmann-Lemaître-Robertson-Walker space-time with non-zero curvature and without the cosmological constant term, and combined with the Refs. [7,27], it is found that if the universe is non-flat (i.e., $k \neq 0$ ), it may also be closed, then the finite equilibrium point $p_{0}$, and the infinite equilibrium points $u_{14}$ and $U_{13}$ can be the late-time states of the universe. Besides Eq. (6) also imply that the value of Hubble parameter $H$ tends to be zero (i.e., the final state is the Minkowski point) in the cosmological model as time goes on.

Acknowledgements The authors thank the anonymous reviewer whose comments and suggestions helped improve and clarify this manuscript. The first author gratefully acknowledges the support of the National Natural Science Foundation of China (NSFC) through Grant Nos. 11672259 and 11571301, the China Scholarship Council through Grant No. 201908320086, the Ministry of Land and Resources Research of China in the Public Interest through Grant No. 201411007.

The second author gratefully acknowledges the support of the Ministerio de Economía, Industria y Competitividad, Agencia Estatal de Investigación grants MTM2016-77278-P (FEDER) and MDM-20140445, the Agència de Gestió d'Ajuts Universitaris i de Recerca grant 2017SGR1617, and the H2020 European Research Council Grant MSCA-RISE-2017-777911.

Data Availability Statement This manuscript has no associated data or the data will not be deposited. [Authors' comment: This manuscript mainly involves qualitative analysis, not quantitative and numerical analysis, so there is no associated data.]

Open Access This article is licensed under a Creative Commons Attribution 4.0 International License, which permits use, sharing, adaptation, distribution and reproduction in any medium or format, as long as you give appropriate credit to the original author(s) and the source, provide a link to the Creative Commons licence, and indicate if changes were made. The images or other third party material in this article are included in the article's Creative Commons licence, unless indicated otherwise in a credit line to the material. If material is not included in the article's Creative Commons licence and your intended use is not permitted by statutory regulation or exceeds the permitted use, you will need to obtain permission directly from the copyright holder. To view a copy of this licence, visit http://creativecomm ons.org/licenses/by/4.0/.

Funded by SCOAP ${ }^{3}$.

\section{References}

1. P. Hořava, Quantum gravity at a Lifshitz point. Phys. Rev. D 79, 084008 (2009)

2. E.M.C. Abreu, A.C.R. Mendes, G. Oliveira-Neto et al., HořavaLifshitz cosmological models with noncommutative phase space variables. Gen. Relat. Gravit. 51, 95 (2019)

3. G. Leon, C.R. Fadragas, Cosmological dynamical systems: and their applications (Lambert Academic Publishing, GmbH \& Co., KG, Saarbrucken, 2012)

4. G. Leon, A. Paliathanasis, Extended phase-space analysis of the Hořava-Lifshitz cosmology. Eur. Phys. J. C 79, 746 (2019)

5. G. Leon, E.N. Saridakis, Phase-space analysis of Hořava-Lifshitz cosmology. J. Cosmol. Astropart. Phys. 2009, 006 (2009)

6. S. Carloni, E. Elizalde, P.J. Silva, An analysis of the phase space of Hořava-Lifshitz cosmologies, in Cosmology, Quantum Vacuum and Zeta Functions, Springer Proceedings in Physics, vol. 137, ed. by S.D. Odintsov, D. Sáez-Gómez, S. Xambó-Descamps (SpringerVerlag, Berlin, 2011), pp. 139-148

7. F.B. Gao, J. Llibre, Global dynamics of the Hořava-Lifshitz cosmological system. Gen. Relat. Gravit. 51(11), 152 (2019)

8. B. Chen, On Hořava-Lifshitz cosmology. Chin. Phys. C 35(5), 429-435 (2011)

9. X. Gao, Y. Wang, R. Brandenberger, A. Riotto, Cosmological perturbations in Hořava-Lifshitz gravity. Phys. Rev. D 81, 083508 (2010)

10. S. Lepe, J. Saavedra, On Hořava-Lifshitz cosmology. Astrophys. Space Sci. 350, 839-843 (2014)

11. M. Li, Y. Pang, A trouble with Hořava-Lifshitz gravity. J. High Energy Phys. 08, 015 (2009)

12. O. Luongo, M. Muccino, H. Quevedo, Kinematic and statistical inconsistencies of Hořava-Lifshitz cosmology. Phys. Dark Univ. 25, 100313 (2019)

13. N.A. Nilsson, E. Czuchry, Hořava-Lifshitz cosmology in light of new data. Phys. Dark Univ. 23, 100253 (2019)

14. A. Paliathanasis, G. Leon, Cosmological solutions in Hořavaifshitz gravity, arXiv preprint arXiv:1903.10821 (2019)

15. E.N. Saridakis, Aspects of Hořava-Lifshitz cosmology. Int. J. Modern Phys. D 20(08), 1485-1504 (2011)

16. A. Tawfik, E. Abou El Dahab, FLRW cosmology with HořavaLifshitz gravity: impacts of equations of state. Int. J. Theoret. Phys. 56(7), 2122-2139 (2017)

17. C.R. Fadragas, G. Leon, E.N. Saridakis, Dynamical analysis of anisotropic scalar-field cosmologies for a wide range of potentials. Classical Quant. Gravity 31, 075018 (2014)

18. M. Bhattacharjee, Gravitational radiation and black hole formation from gravitational collapse in theories of gravity with broken Lorentz symmetry, Baylor University. ProQuest Dissertations Publishing 22585106 (2019) 
19. S. Mukohyama, Hořava-Lifshitz cosmology: a review. Classical Quant. Gravity 27, 223101 (2010)

20. T.P. Sotiriou, Hořava-Lifshitz gravity: a status report. J. Phys. Conf. Series 283, 012034 (2011)

21. E. Kiritsis, G. Kofinas, Hořava-Lifshitz cosmology. Nuclear Phys. B 821, 467-480 (2009)

22. D. Escobar, C.R. Fadragas, G. Leon, Y. Leyva, Asymptotic behavior of a scalar field with an arbitrary potential trapped on a RandallSundrum's braneworld: the effect of a negative dark radiation term on a Bianchi I brane. Astrophys. Space Sci. 349, 575-602 (2014)

23. A. Alho, J. Hell, C. Uggla, Global dynamics and asymptotics for monomial scalar field potentials and perfect fluids. Classical Quant. Gravity 32, 145005 (2015)
24. F. Dumortier, J. Llibre, J.C. Artés, Qualitative Theory of Planar Differential Systems (Springer-Verlag, Berlin, 2006)

25. A. Cima, J. Llibre, Bounded polynomial vector fields. Trans. Am. Math. Soc. 318(2), 557-579 (1990)

26. M.J. Álvarez, A. Ferragut, X. Jarque, A survey on the blow up technique. Int. J. Bifurc. Chaos 21(11), 3103-3118 (2011)

27. E. Di Valentino, A. Melchiorri, J. Silk, Planck evidence for a closed universe and a possible crisis for cosmology. Nat. Astron. (2019). https://doi.org/10.1038/s41550-019-0906-9 